\title{
MINI-REVIEW ON COLLIDER SIGNATURES FOR EXTRA DIMENSIONS
}

\author{
KINGMAN CHEUNG \\ Department of Physics, University of California, Davis, CA 95616 USA \\ E-mail: cheung@gluon.ucdavis.edu
}

\begin{abstract}
In this talk, I briefly review collider signatures for two models of extra dimensions. The first one was proposed by Arkani-Hamed et al. that gravity is free to propagate in extra dimensions of very large size $(\lesssim 1 \mathrm{~mm})$. Collider signatures for this model can be divided into two types: (i) emission of real gravitons into extra dimensions, and (ii) exchanges of virtual gravitons. The second model was proposed by Pomarol et al. and Dienes et al. that the SM gauge bosons are allowed to propagate in extra dimensions. Collider signatures for the second model are due to the existence of the Kaluza-Klein (KK) states of $\gamma, W, Z$, and $g$ bosons.
\end{abstract}

\section{Introduction}

Recent advances in string theory have revolutionized particle phenomenology. Namely, the previously unreachable Planck, string, and grand unification scales $\left(M_{\mathrm{Pl}}, M_{\mathrm{st}}\right.$, and $M_{\mathrm{GUT}}$, respectively) can be brought down to a $\mathrm{TeV}$ range through the existence of extra dimensions. There have been a number of ideas that can bring either the Planck, GUT, or string scale down to $\mathrm{TeV}$ region. One expects the low energy phenomenology of these new ideas or models can be tested at current and future collider experiments.

In this talk, we review collider signatures for the following two models of extra dimensions: (i) the one proposed by Arkani-Hamed, Dimopoulos, and Dvali, in which the standard model (SM) particles live on a 3-brane while the gravity is free to propagate in extra dimensions of very large size $(\lesssim 1 \mathrm{~mm})$. This model was motivated by the fact that the effective Planck scale is brought down to $\mathrm{TeV}$ to solve the hierarchy problem. Collider signatures for this model can be divided into two types: (a) emission of real gravitons into extra dimensions and thus gives rise to missing energy signals, and (b) exchanges of virtual gravitons that frequently lead to enhancement of production of SM particles. (ii) The model proposed by Dienes et al. 3 and Pomarol et al.3 that the SM gauge bosons are allowed to propagate in extra dimensions (whereas the gravity effect here is negligible.) This model has the merit of unifying the gauge couplings at a scale much lower than the usual GUT scale. Collider signatures for this model are due to the existence of the Kaluza-Klein (KK) states of $\gamma, W, Z$, and $g$ bosons.

\section{Model of Arkani-Hamed, Dimopoulos, and Dvali}

This model was first proposed to solve the hierarchy problem by requiring the compactified dimensions to be of very large size, $\lesssim 1 \mathrm{~mm}$. While the SM particles live on a D3-brane, the gravity is free to propagate in extra dimensions. Using Gauss law, the effective Planck scale $M_{S}$ is related to the four-dimensional Planck scale $M_{\mathrm{Pl}}\left(10^{19} \mathrm{GeV}\right)$ by

$$
M_{\mathrm{Pl}}^{2} \sim M_{S}^{n+2} R^{n}
$$

where $n$ is the number of extra (compactified) dimensions and $R$ is the size of the compactified dimensions. Assuming that the effective Planck scale $M_{S}$ is in the TeV range, it gives a very large $R$ of the size of a solar system for $n=1$, which is obviously ruled out by experiments. However, for all $n \geq 2$ the expected $R$ is less than $1 \mathrm{~mm}$, and therefore do not contradict existing gravitational experiments. 
With the SM particles residing on the brane and the graviton freely propagating in extra dimensions, in the $4 \mathrm{D}$-point of view a graviton in extra dimensions is equivalent to a tower of infinite number of KK states with masses $M_{k}=2 \pi k / R \quad(k=0,1,2, \ldots, \infty)$. The couplings of SM particles to each of these KK states is still of order $1 / M_{\mathrm{Pl}}$, but the overall coupling is, however, obtained by summing over all the KK states, and therefore scales as $1 / M_{S}$. Since the $M_{S}$ is in the TeV range, the effective gravitational interaction is as strong as the electroweak interaction and thus gives rise to many testable consequences in both accelerator and non-accelerator experiments.

A large number of phenomenological studies 45 in this area have appeared. The collider signatures can be divided into two categories: (i) real emission of gravitons into extra dimensions, giving rise to missing energy signal, and (ii) virtual exchange of gravitons in addition to exchanges of SM gauge bosons, giving rise to enhancement or deviations from the SM predictions. We summarize these signals in the following. Note that a stringent constraint comes from astrophysical (SN1987A) and cosmological sources $\exists$ and the lower bound on the effective Planck scale $M_{S}$ is $30-100 \mathrm{TeV}$ for $n=2$.

\subsection{Real Emission of Gravitons}

Since gravitons interact weakly with detectors, they will escape detection and causing missing energies. Thus, the logical signal to search for would be the associated production of gravitons with other SM particles. At $e^{+} e^{-}$colliders, the best signals would be the associated production of graviton with a $Z$ boson, a photon, or a fermion pair.

The production of graviton and photon at LEPII has been studied. 1.613 The striking signature would be a single photon with missing energy while the irreducible backgrounf comes from the process $e^{+} e^{-} \rightarrow \gamma \nu_{\ell} \bar{\nu}_{\ell},(\ell=e, \mu, \tau)$. The cross section for the signal is given by $5,6,13$

$$
\begin{aligned}
\frac{d \sigma}{d \cos \theta} & =\frac{\pi \alpha G_{N}}{4\left(1-\frac{m^{2}}{s}\right)}\left[\left(1+\cos ^{2} \theta\right)\left(1+\left(\frac{m^{2}}{s}\right)^{4}\right)\right. \\
& \left.+\left(\frac{1-3 \cos ^{2} \theta+4 \cos ^{4} \theta}{1-\cos ^{2} \theta}\right) \frac{m^{2}}{s}\left(1+\left(\frac{m^{2}}{s}\right)^{2}\right)+6 \cos ^{2} \theta\left(\frac{m^{2}}{s}\right)^{2}\right] .
\end{aligned}
$$

The signal cross section increases with the energy of collision while the background is gradually decreasing after the $Z$-peak. At LEPII, if the effective Planck scale $M_{S}$ is low enough deviations from the SM prediction should be seen. In a recent search by L3,55 the limit $M_{S} \gtrsim 1 \mathrm{TeV}$ for $n=2$.

The production of graviton with a $Z$ boson at LEPI 3 gives a signature of a $Z$ boson, which decays into a $q \bar{q}$ or $\ell \bar{\ell}$ pair, plus missing energy. This is an interesting process because LEPII already searched for the invisibly decaying Higgs boson in $Z H$ production, which has the same signature as the $Z G$ production. The formulas for the signal cross section can be found in Ref.13 The irreducible background is $e^{+} e^{-} \rightarrow Z \nu \bar{\nu}$. Based on the existing data on the search for invisibly decaying Higgs, the lower limit on $M_{S}$ is around $515 \mathrm{GeV}$. A refined search by L355 gives a limit $M_{S}>600 \mathrm{GeV}$.

The associated production of graviton with a $f \bar{f}$ pair was studied at $Z^{016}$ because of a large number of hadronic $Z$ decays. The signature would be a fermion pair with missing energy. The background comes from $Z \rightarrow f \bar{f} \nu \bar{\nu}$, which has a small BR of $2 \cdot 10^{-7}$. The present data agrees with the SM prediction and is able to constrain $M_{S}$ to be at least $0.4 \mathrm{TeV}$ for $n=2.16$

Another exciting opportunity is the monojet plus missing energy production 1 t at hadronic colliders.

summary: submitted to World Scientific on November 2, 2018 


\subsection{Virtual Exchanges of gravitons}

There are numerous studies in collider signatures associated with virtual exchanges of KK-gravitons, including diphoton, diboson, and fermion-pair production. In the following, we highlight some of these studies.

One of the most prominent channels is photon-photon scattering. In the SM, photon-photon scattering only takes place via box diagrams of $W$ bosons and quarks so that it is loop-suppressed. On the other hand, in the ADD model photons can scatter via exchanges of gravitons in $s, t, u$-channels. The scattering amplitude-squared is symmetric in $s, t, u$ and given by $3 \bar{\sum}|\mathcal{M}|^{2}=\frac{\kappa^{4}}{8}|D(s)|^{2}\left(s^{4}+\right.$ $\left.t^{4}+u^{4}\right)$. The differential cross section is given by

$$
\frac{d \sigma(\gamma \gamma \rightarrow \gamma \gamma)}{d|\cos \theta|}=\frac{\pi s^{3}}{M_{S}^{8}} \mathcal{F}^{2}\left[1+\frac{1}{8}\left(1+6 \cos ^{2} \theta+\cos ^{4} \theta\right)\right]
$$

where $\mathcal{F}=2 /(n-2)$ for $n>2$. The signal cross section easily surpasses the SM background at around $\sqrt{s}=0.5 \mathrm{TeV}$ for $M_{S}=4 \mathrm{TeV} 36$ The polarized scattering has also been studied in Ref. 19.20 .52 Another interesting channel is neutrino-photon scattering. 54

Diphoton production 13 and also $W W, Z Z$ production 11 at $e^{+} e^{-}$colliders have been studied. The effect of $\mathrm{TeV}$ scale gravity on the angular distribution of diphoton production $B \mathrm{E}$ is

$$
\frac{d \sigma\left(e^{+} e^{-} \rightarrow \gamma \gamma\right)}{d z}=\frac{2 \pi}{s}\left(\alpha \sqrt{\frac{1+z^{2}}{1-z^{2}}}+\frac{s^{2}}{8} \frac{\mathcal{F}}{M_{S}^{4}} \sqrt{1-z^{4}}\right)^{2}
$$

where $z=\left|\cos \theta_{\gamma}\right|$. This effect is very similar to the conventional deviation from QED, which is often parametrized by a $\Lambda$ as

$$
\frac{d \sigma}{d z}=\frac{2 \pi \alpha^{2}}{s} \frac{1+z^{2}}{1-z^{2}}\left(1 \pm \frac{s^{2}}{2 \Lambda_{ \pm}^{4}}\left(1-z^{2}\right)\right) .
$$

We can immediately equate the above two expressions and arrive at $\frac{M_{S}^{4}}{\mathcal{F}}=\frac{\Lambda_{+}^{4}}{2 \alpha}$. The present limits obtained by LEP Collaborations on $\Lambda_{\mathrm{QED}} \sim 262-345 \mathrm{GeV}$, which is equivalent to $M_{S} \sim 0.7-1$ $\mathrm{TeV}$ for $n=4.36$

Agashe and Deshpande11 also studied $e^{+} e^{-} \rightarrow \gamma \gamma, W^{+} W^{-}, Z Z$ production and compared their sensitivities to $\mathrm{TeV}$ scale gravity. Interestingly, $Z Z$ production offers the highest fractional change of cross section among $\gamma \gamma, W W, Z Z$ due to gravity effects. However, the $Z Z$ production rate is smaller than the gther two. Overall, their sensitivities are similar. A recent experimental search performed by $\mathrm{L}^{5} 5$ found that the sensitivities of $\gamma \gamma, W W, Z Z$ are very similar and the combined limit is $M_{S} \gtrsim 0.8 \mathrm{TeV}$.

Diphoton production $1,46,41$ is one of the best probes of $\mathrm{TeV}$ scale gravity at hadron colliders. The angular distributions of the subprocesses are given by

$$
\begin{aligned}
& \frac{d \sigma(q \bar{q} \rightarrow \gamma \gamma)}{d \cos \theta^{*}}=\frac{1}{48 \pi \hat{s}}\left[e^{2} Q_{q}^{2} \sqrt{\frac{1+\cos ^{2} \theta^{*}}{1-\cos ^{2} \theta^{*}}}+\frac{\pi \hat{s}^{2}}{2} \frac{\mathcal{F}}{M_{S}^{4}} \sqrt{1-\cos ^{4} \theta^{*}}\right]^{2}, \\
& \frac{d \sigma(g g \rightarrow \gamma \gamma)}{d \cos \theta^{*}}=\frac{\pi \hat{s}^{3}}{512}\left(\frac{\mathcal{F}}{M_{S}^{4}}\right)^{2}\left(1+6 \cos ^{2} \theta^{*}+\cos ^{4} \theta^{*}\right),
\end{aligned}
$$

where $\cos \theta^{*}$ is the scattering angle of the photon in the center-of-mass frame of the incoming partons, and here $\cos \theta^{*}$ is from -1 to 1 . Based on the existing diphoton data from CDF 56 that in $M_{\gamma \gamma}>150$ $\mathrm{GeV} 5$ events are observed where $4.5 \pm 0.6$ are expected with a luminosity of $100 \mathrm{pb}^{-1}$, a limit of $M_{S}>0.9 \mathrm{TeV} 36$ for $n=4$ was obtained. The upcoming CDF and D0 searches will easily overshadow this limit. 
Table 1. $95 \%$ C.L. sensitivity limits on $M_{S}(n=4)$ at (a) hadronic colliders (Tevatron and LHC) and (b) $e^{+} e^{-}$colliders of $0.5-1.5 \mathrm{TeV}$.

\begin{tabular}{|c|c|c|c|c|c|c|c|c|c|c|}
\hline \multicolumn{4}{|c|}{ (a) Hadronic Colliders } & \multicolumn{7}{|c|}{ (b) $e^{+} e^{-}$Colliders } \\
\hline$\overline{\overline{\text { Run I }}}$ & 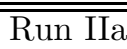 & $\overline{\text { Run IIb }}$ & $\overline{\mathrm{LHC}}$ & $\bar{~} \sqrt{s}=0.5$ & & & $\overline{\overline{1 \mathrm{Te}}}$ & & & \\
\hline $\mathcal{L}=0.13 \mathrm{fb}^{-1}$ & 2 & 20 & 100 & $\mathcal{L}=10 \mathrm{fb}^{-1}$ & 50 & 10 & 50 & 100 & 100 & 200 \\
\hline 1.3 & 1.9 & 2.6 & 9.9 & 3.9 & 4.8 & 6.5 & 7.9 & 8.9 & 12.0 & 13.0 \\
\hline
\end{tabular}

The general vector-boson scattering $V V \rightarrow V V$, where $V=\gamma, W, Z$ was studied by Atwood et al. 2 The conclusion is that the effect of $\mathrm{TeV}$ scale gravity shows up at large invariant mass region.

Extra dimensions also affect fermion-pair production at $e^{+} e^{-}$colliders and the corresponding crossing channels, such as Drell-Yan production at the Tevatron and neutral-current (NC) deepinelastic scattering (DIS) at HERA. While they were individually studied in a number of publications, 3 , 20, 35,43 a comphrensive analysis of all these data sets was performed in Ref. 39 The combined limit on $M_{S}$ is $M_{S}>0.94 \mathrm{TeV}$ for $n=4$. Bourilkov 37 on the other hand, used the combined data of Bhabha scattering of the four LEP Collaborations and was able to obtain a limit of $M_{S}>1.4$ $\mathrm{TeV}$. There are also a combined search in fermion-pair production, diphoton, $W W$ and $Z Z$ pair production by $\mathrm{L} 355$ that a limit of $\mathrm{M}_{\mathrm{S}}>\mathrm{TeV}$ is established.

Dijet and top-pair production 12.445 at the Tevatron or other colliders should also be useful in obtaining information on $M_{S}$, but systematics will likely reduce the usefulness of these channels, Effects on precision variables, 12,2253 and effects on patterns of fermion or neutrino masses 21 27. 30. 31 have also been studied. Interactions with scalars or Higgs bosons have been investigated in Ref.14.23.25, 45

In the following, we describe a few studies that test sensitivity to $M_{S}$ in future experiments at hadronic and $e^{+} e^{-}$colliders. Cheung and Landsberg 40 used double differential cross-sections, $d^{2} \sigma / d M d \cos \theta^{*}$, of diphoton and Drell-Yan production to constrain the effective Planck scale $M_{S}$ in Run I and Run II at the Tevatron and at the LHC. The advantage of using double differential distributions is that the differences in invariant mass and scattering angle between the SM and the gravity model can be contrasted simultaneously. Furthermore, for a $2 \rightarrow 2$ process the invariant mass $M$ and the central scattering angle $\cos \theta^{*}$ already span the entire phase space. We, therefore, do not need further optimization of cuts or variables. The resulting sensitivities to $M_{S}$ are shown in Table 11(a).

The sensitivity reach at linear $e^{+} e^{-}$colliders was studied in a number of work. Here I present a work $\mathbb{E}^{-}$that uses diphoton, Bhabha scattering, $\mu^{+} \mu^{-}, \tau^{+} \tau^{-}, q \bar{q}$ production and their angular distributions. The sensitivity limits on $M_{S}$ are obtained by a combined maximum likelihood approach by adding the likelihoods of all channels. The Bhabha scattering turns out to be the dominant channel. The angular distribution for diphoton production is given in Eq. (2) and for fermion-pair production it is given by

$$
\begin{aligned}
& \frac{d \sigma\left(e^{-} e^{+} \rightarrow f \bar{f}\right)}{d z}=\frac{N_{f} s}{128 \pi}\left\{(1+z)^{2}\left(\left|M_{L L}(s)\right|^{2}+\left|M_{R R}(s)\right|^{2}\right)+(1-z)^{2}\right. \\
& \quad \times\left(\left|M_{R L}(s)\right|^{2}+\left|M_{L R}(s)\right|^{2}\right)+\pi^{2} s^{2}\left(1-3 z^{2}+4 z^{4}\right) \eta^{2} \\
& \left.\quad-8 \pi e^{2} Q_{e} Q_{f} z^{3} \eta+\frac{8 \pi e^{2}}{s_{\theta}^{2} c_{\theta}^{2}} \frac{s}{s-M_{Z}^{2}}\left(g_{a}^{e} g_{a}^{f} \frac{1-3 z^{2}}{2}-g_{v}^{e} g_{v}^{f} z^{3}\right) \eta\right\}
\end{aligned}
$$

summary: submitted to World Scientific on November 2, 2018 


$$
\begin{aligned}
& +\frac{\delta_{e f} s}{128 \pi}\left\{( 1 + z ) ^ { 2 } \left(\left|M_{L L}(t)\right|^{2}+\left|M_{R R}(t)\right|^{2}+2 M_{L L}(s) M_{L L}(t)\right.\right. \\
& \left.+2 M_{R R}(s) M_{R R}(t)\right)+4\left(\left|M_{L R}(t)\right|^{2}+\left|M_{R L}(t)\right|^{2}\right)+\frac{\pi^{2} s^{2}}{8} \\
& \times\left(121+228 z+198 z^{2}+84 z^{3}+9 z^{4}\right) \eta^{2}-\frac{\pi s}{2} \eta\left(M_{L L}(t)+M_{R R}(t)\right. \\
& \left.+M_{L L}(s)+M_{R R}(s)\right)(1+z)^{2}(7+z)+\pi s \eta\left(M_{L L}(t)+M_{R R}(t)\right) \\
& \left.\times(1+z)^{2}(1-2 z)-2 \pi s \eta\left(M_{L R}(t)+M_{R L}(t)\right)(5+3 z)\right\}
\end{aligned}
$$

where $\eta=\mathcal{F} / M_{S}^{4}$ and the reduced amplitudes are given by

$$
M_{\alpha \beta}^{e f}(s)=\frac{e^{2} Q_{e} Q_{f}}{s}+\frac{e^{2}}{\sin ^{2} \theta_{\mathrm{w}} \cos ^{2} \theta_{\mathrm{w}}} \frac{g_{\alpha}^{e} g_{\beta}^{f}}{s-M_{Z}^{2}}, \quad \alpha, \beta=L, R .
$$

The $95 \%$ C.L. sensitivity limits on $M_{S}$ for $n=4$ are shown in Table 1 1 (b). The values for other $n$ can be easily obtained by scaling with $\mathcal{F}=2 /(n-2)$ for $n>2$. From the table we can see that a $0.5 \mathrm{TeV}$ NLC has a reach at least double of that at RunIIa and still higher than that of RunIIb. The reach by the LHC is only slightly better than the $1 \mathrm{TeV}$ NLC.

\section{Kaluza-Klein States of SM Gauge Bosons}

In another brane configuration, the SM particles reside on a $p$-brane $(p=\delta+3>3)$ whereas the gravity is in the rest $(6-p)$ dimensional bulk. Within this $p$-brane the effect of gravity is negligible compared to gauge interactions. Inside the $p$-brane the chiral fermions are restricted to a 3-brane and the gauge bgsons can propagate in the extra $\delta$ dimensions internal to the $p$-brane.

Dienes et al.2 considered in this model of extra dimensions and showed that the gauge couplings can be unified at a scale lower than the usual GUT scale, due to the power running of the couplings. Therefore, the effective GUT scale is lowered, which is in contrast to the ADD model that the Planck scale is lowered.

For collider phenomenology a specific model of this type was proposed by Pomarol et al . $^{\text {It is }}$ a five-dimensional model with the fifth dimension compactified on a $S^{1} / Z_{2}$. In the four-dimensional point of view, a gauge boson $V$ that propagates in the fifth dimension is equivalent to a tower of Kaluza-Klein states $V^{(n)}$ with mass $M_{n}=n M_{c}$, where $M_{c}=1 / R$ is the compactification scale and $R$ is radius of the fifth dimension. The resulting 4-D Lagrangian for charged-current and neutralcurrent interactions are, respectively, given by

$$
\begin{aligned}
\mathcal{L}^{\mathrm{CC}} & =\frac{g^{2} v^{2}}{8}\left[W_{1}^{2}+\cos ^{2} \beta \sum_{n=1}^{\infty}\left(W_{1}^{(n)}\right)^{2}+2 \sqrt{2} \sin ^{2} \beta W_{1} \sum_{n=1}^{\infty} W_{1}^{(n)}+2 \sin ^{2} \beta\right. \\
& \left.\times\left(\sum_{n=1}^{\infty} W_{1}^{(n)}\right)^{2}\right]+\frac{1}{2} \sum_{n=1}^{\infty} n^{2} M_{c}^{2}\left(W_{1}^{(n)}\right)^{2}-g\left(W_{1}^{\mu}+\sqrt{2} \sum_{n=1}^{\infty} W_{1}^{(n) \mu}\right) J_{\mu}^{1} \\
& +(1 \rightarrow 2) \\
\mathcal{L}^{\mathrm{NC}} & =\frac{g v^{2}}{8 c_{\theta}^{2}}\left[Z^{2}+\cos ^{2} \beta \sum_{n=1}^{\infty}\left(Z^{(n)}\right)^{2}+2 \sqrt{2} \sin ^{2} \beta Z \sum_{n=1}^{\infty} Z^{(n)}+2 \sin ^{2} \beta\right.
\end{aligned}
$$




$$
\begin{aligned}
& \times\left(\sum_{n=1}^{\infty} Z^{(n)}\right)^{2}+\frac{1}{2} \sum_{n=1}^{\infty} n^{2} M_{c}^{2}\left[\left(Z^{(n)}\right)^{2}+\left(A^{(n)}\right)^{2}\right] \\
& -\frac{e}{s_{\theta} c_{\theta}}\left(Z^{\mu}+\sqrt{2} \sum_{n=1}^{\infty} Z^{(n) \mu}\right) J_{\mu}^{Z}-e\left(A^{\mu}+\sqrt{2} \sum_{n=1}^{\infty} A^{(n) \mu}\right) J_{\mu}^{\mathrm{em}}
\end{aligned}
$$

There are two important effects of these KK states on collider experiments. (i) Since the KK states have the same quantum numbers as their corresponding gauge bosons, it gives rise to mixing effects between the zeroth (the SM gauge boson) and the $n$ th-modes of $W$ and $Z$ bosons. The zero mass of the photon is protected by the U(1) EM symmetry of the SM. (ii) If the energy is higher than the compactification scale $M_{c}$, real emissions or resonances of KK states of $\gamma, W, Z, g$ bosons can be observed, otherwise enhancement of cross sections may be possible.

In Eqs. (5) and (6) the first few terms of each imply mixings among $V, V^{(1)}, V^{(2)}, \ldots(V=$ $W, Z)$. The mixing between the SM gauge bosons with its Kaluza-Klein states modifies electroweak observables (similar to the mixing between the $Z$ and a $Z^{\prime}$ ) via a series of mixing angles, which depend on the masses of $Z^{(n)}, n=0,1, \ldots$ and the angle $\beta$. The neutral boson at LEP is then the first mass eigenstate after mixing. The couplings of the $Z^{(0)}$ to fermions are modified through the mixing angles. The observables at LEPI can place strong constraints on the mixing, and thus on the compactification scale $M_{c}$. Similarly, the properties of the $W$ boson are also modified. The effects

on electroweak precision measurements have been studied.58 64 Overall, the limit on $M_{c}$ using the precision data measurements is $M_{c} \gtrsim 3.3-3.8 \mathrm{TeV}$.

The effects of Kaluza-Klein states of the SM gauge bosons also occur in high energy processes. If the available energy is higher than the compactification scale, real emissions or resonances of these Kaluza-Klein states can be observed. However, for the present collider energies and because the compactification scale is believed to be at least a few $\mathrm{TeV}$, only indirect effects can be seen.

We summarize a study of high energy processes when $M_{c}$ is higher than the present energy scale. The reduced amplitude for $q \bar{q} \rightarrow \ell^{+} \ell^{-}$or $\ell^{+} \ell^{-} \rightarrow q \bar{q}$ is given by

$$
M_{\alpha \beta}^{e q}(s)=e^{2}\left\{\frac{Q_{e} Q_{q}}{s}+\frac{g_{\alpha}^{e} g_{\beta}^{q}}{s_{\theta}^{2} c_{\theta}^{2}} \frac{1}{s-M_{Z}^{2}}-\left(Q_{e} Q_{q}+\frac{g_{\alpha}^{e} g_{\beta}^{q}}{s_{\theta}^{2} c_{\theta}^{2}}\right) \frac{\pi^{2}}{3 M_{c}^{2}}\right\} .
$$

where $M_{c}^{2} \gg s,|t|,|u|$. The above formula is applicable to hadronic and leptonic cross sections at $e^{+} e^{-}$colliders and to Drell-Yan production at the Tevatron, and with a crossing to DIS at HERA. Similar expressions can be found 65 for $W$ KK state exchanges and for dijet and $t \bar{t}$ production. In a global fit to $\eta=\pi^{2} / 3 M_{c}^{2}$, Cheung and Landsberg65 include the following data sets: (i) LEPII hadronic and all leptonic production cross sections and angular distributions, (ii) Drell-Yan production at the Tevatron, (iii) NC and CC DIS scattering cross sections at HERA, (iv) Tevatron dijet production, and (v) Tevatron $t \bar{t}$ production. The resulting 95\% C.L. limit on $M_{c}$ is

$$
M_{c}>4.4 \mathrm{TeV} \text {. }
$$

\section{Conclusions}

Physics in extra dimensions and phenomenology are extremely rich with advance in string theories because fundamental scales are now reachable within future collider experiments. We have briefly reviewed collider signatures of two interesting scenarios. The first model is due to the KK states of gravitons while the second one is due to KK states of gauge bosons. It turns out that diphoton, boson-pair, and fermion-pair production, as well as precision data measurements are useful in probing these two models. 


\section{Acknowledgments}

I thank Wai-yee Keung and Greg Landsberg for parts of the work presented here. This research was supported in part by the DOE Grants No. DE-FG03-91ER40674.

\section{References}

1. N. Arkani-Hamed, S. Dimopoulos, G. Dvali, Phys. Lett. B429, 263 (1998); I. Antoniadis, N. Arkani-Hamed, S. Dimopoulos, and G. Dvali, Phys. Lett. B436, 257 (1998); N. Arkani-Hamed, S. Dimopoulos, G. Dvali, Phys. Rev. D 59, 086004 (1999);

2. K. Dienes, E. Dudas, and T. Gherghetta, Nucl. Phys. B537, 47 (1999).

3. A. Pomarol and M. Quirós, Phys. Lett. B438, 255 (1998); M. Masip and A. Pomarol, Phys. Rev. D60, 096005 (1999); I. Antoniadis, K. Benakli, and M. Quirós, Phys. Lett. B460, 176 (1999).

4. S. Cullen and M. Perelstein, Phys. Rev. Lett. 83, 268 (1999); L. Hall and D. Smith, Phys. Rev. D60, 085008 (1999); V. Barger et al., Phys. Lett. B461, 34 (1999);

5. G. Giudice, R. Rattazzi, and J. Wells, Nucl. Phys. B544, 3 (1999).

6. S. Nussinov and R. Shrock, Phys. Rev. D 59, 105002 (1999).

7. E. Mirabelli, M. Perelstein, and M. Peskin, Phys. Rev. Lett. 82, 2236 (1999).

8. T. Han, J. Lykken, and R. Zhang, Phys. Rev. D 59, 105006 (1999).

9. J. Hewett, Phys. Rev. Lett. 82, 4765 (1999).

10. T. Rizzo, Phys. Rev. D 59, 115010 (1999).

11. K. Agashe and N. Deshpande, Phys. Lett. B456, 60 (1999).

12. M. Graesser, Phys. Rev. D61, 074019 (2000).

13. K. Cheung and W.-Y. Keung, Phys. Rev. D60, 112003 (1999).

14. T. Rizzo, Phys. Rev. D 60, 075001 (1999).

15. D. Atwood, S. Bar-Shalom, and A. Soni, hep-ph/9903538.

16. C. Balázs et al., Phys. Rev. Lett. 83, 2112 (1999).

17. G. Shiu, R. Shrock, and S. Tye, Phys.Lett. B458, 274 (1999).

18. K. Lee, H. Song, J. Song, Phys. Lett. B464, 82 (1999).

19. T. Rizzo, Phys. Rev. D60, 115010 (1999).

20. H. Davoudiasl, Phys. Rev. D60, 084022 (1999).

21. K. Yoshioka, KUNS-1569, e-Print Archive: hep-ph/9904433;

22. K. Lee et al., Phys. Rev. D60, 093002 (1999).

23. X. He, Phys. Rev. D60, 115017 (1999).

24. T. Han, D. Rainwater, and D. Zeppenfeld, Phys. Lett. B463, 93 (1999).

25. X. He, Phys. Rev. D61, 036007 (2000).

26. A. Pilaftsis, Phys. Rev. D60, 105023 (1999).

27. A. Das and O. Kong, Phys. Lett. B470, 149 (1999).

28. Z.K. Silagadze, hep-ph/9907328;

29. H. Davoudiasl, Phys. Rev. D61, 044018 (2000).

30. R.N. Mohapatra, S. Nandi, A. Perez-Lorenzana, Phys.Lett. B466, 115 (1999).

31. A. Ioannisian and A. Pilaftsis, hep-ph/9907522.

32. P. Das and S. Raychaudhuri, hep-ph/9908205.

33. Z.K. Silagadze, Mod. Phys. Lett.A14, 2321 (1999).

34. H.C. Cheng and K. Matchev, Nucl. Phys. B563, 21 (1999).

35. A. Gupta, N. Mondal, and S. Raychaudhuri, hep-ph/9904234:

36. K. Cheung, Phys. Rev. D61, 015005 (2000).

summary: submitted to World Scientific on November 2, 2018 
37. D. Bourilkov, JHEP 9908:006,1999

38. D. Atwood, S. Bar-Shalom, and A. Soni, hep-ph/9906400;

39. K. Cheung, Phys. Lett. B460, 383 (1999).

40. K. Cheung and G. Landsberg, hep-ph/9909218.

41. O.J.P. Éboli et al., hep-ph/9908358.

42. P. Mathews, S. Raychaudhuri, and K. Sridhar, Phys. Lett. B450, 343 (1999).

43. P. Mathews, S. Raychaudhuri, and K. Sridhar, Phys. Lett. B455, 115 (1999)

44. P. Mathews, S. Raychaudhuri, and K. Sridhar, hep-ph/9904232.

45. B. Grzadkowski and J. Gunion, Phys. Lett. B473, 50-60 (2000).

46. K. Lee et al., Phys. Rev. D61, 074005 (2000).

47. D. Ghosh, P. Poulose, and K. Sridhar, hep-ph/9909377.

48. D. Atwood, S. Bar-Shalom, and A. Soni, hep-ph/9909392.

49. D. Atwood, S. Bar-Shalom, and A. Soni, hep-ph/9911231.

50. M. Doncheski and R. Robinett, hep-ph/9910346

51. D. Ghosh, P. Mathews, P. Poulose, and K. Sridhar, JHEP 9911:004 (1999).

52. S. Choudhury, A. Cornell, and G. Joshi, hep-ph/0001061.

53. T. Han, D. Marfatia, and R. Zhang, hep-ph/0001320

54. D. Dicus, K. Kovner, and W. Repko, hep-ph/0003152.

55. L3 Coll., Phys. Lett. B464, 135 (1999); Phys. Lett. B470, 281 (1999).

56. Submission by P. Wilson, CDF Coll., to ICHEP'98.

57. Talk presented by K. Cheung at Berkeley 2000, LBL, Berkeley. Slides can be found at http://gluon.ucdavis.edu/ ${ }^{\sim}$ cheung/berkeley2000.ps.

58. P. Nath and M. Yamaguchi, Phys. Rev. D60, 116004 (1999); P. Nath, Y. Yamada, and M.

Yamaguchi, Phys. Lett. B466, 100 (1999).

59. T. Rizzo and J. Wells, Phys. Rev. D61, 016007 (2000).

60. F. Cornet, M. Relaño, and J. Rico, Phys. Rev. D61, 037701 (2000).

61. R. Casalbouoni, et al. Phys. Lett. B462, 48 (1999).

62. A. Strumia, Phys. Lett. B466, 107 (1999).

63. C. Carone, Phys. Rev. D61, 015008 (2000).

64. A. Delgado, A. Pomarol, and M. Quiros, JHEP 0001:030 (2000).

65. K. Cheung and G. Landsberg, in preparation. 